\title{
Correction to: Integrating STEM into Preschool Education; Designing a Professional Development Model in Diverse Settings
}

\author{
Kimberly Brenneman ${ }^{1,3} \cdot$ Alissa Lange $^{2} \cdot$ Irena Nayfeld $^{2}$ \\ Published online: 1 November 2018 \\ (c) Springer Nature B.V. 2018 \\ Correction to: Early Childhood Education Journal \\ https://doi.org/10.1007/s10643-018-0912-z
}

The original version of this article unfortunately contained a mistake in Funding section. Some of the vital information is missing in the published article. The complete funding information is presented with this erratum.

Funding Work presented here is made possible by grants from the National Science Foundation (DRL-1019576 \& DRL-1417040/DRL-1726082). Any opinions, findings, and conclusions or recommendations expressed in this material are those of the author(s) and do not necessarily reflect the views of the National Science Foundation.

The original article can be found online at https://doi.org/10.1007/ s10643-018-0912-z.

Irena Nayfeld

inayfeld@gmail.com

1 National Institute for Early Education Research, Rutgers University, 73 Easton Ave, New Brunswick, NJ 08901, USA

2 Department of Early Childhood Education, East Tennessee State University, Johnson City, TN 37614, USA

3 Present Address: Heising-Simons Foundation, 400 Main Street, Suite 200, Los Altos, CA 94022, USA 